\title{
Effect of fibroblast growth factor-2 on periodontal ligament cells derived from human deciduous teeth in vitro
}

\author{
TOMOKAZU HASEGAWA ${ }^{1}$, NAOYUKI CHOSA ${ }^{2}$, TAKEYOSHI ASAKAWA ${ }^{1}$, YOSHITAKA YOSHIMURA ${ }^{3}$, \\ ASAMI ASAKAWA ${ }^{1}$, AKIRA ISHISAKI ${ }^{2}$ and MITSURO TANAKA ${ }^{1}$
}

Departments of ${ }^{1}$ Pediatric Dentistry, and ${ }^{2}$ Oral Biochemistry, School of Dentistry, Iwate Medical University, Morioka 020-8505; ${ }^{3}$ Department of Molecular Cell Pharmacology, Division of Oral Pathological Science, Hokkaido University Graduate School of Dental Medicine, Sapporo 060-8586, Japan

Received November 12, 2009; Accepted December 21, 2009

DOI: 10.3892/etm_00000052

\begin{abstract}
A blood supply is crucial for tissue healing and regeneration. Periodontal ligament (PDL) tissue is situated between the tooth root and alveolar bone, and cells derived from PDL tissue are reported to have stem cell-like activity. This study aimed to evaluate the potential of PDL cells derived from deciduous teeth to express endothelial cell (EC)-specific markers. Using quantitative PCR, we investigated whether PDL cells derived from human deciduous teeth express mRNA for the EC-specific markers: vascular endothelialcadherin (VE-cadherin), vascular endothelial growth factor receptor 2 (VEGFR2) and CD31 upon treatment with $15 \mathrm{ng} / \mathrm{ml}$ heparin or $10 \mathrm{ng} / \mathrm{ml}$ fibroblast growth factor (FGF)-2 in vitro. Quantitative PCR showed that PDL cells expressed mRNA for the EC-specific markers, VE-cadherin and VEGFR2, when cultured in the presence of heparin alone or with FGF-2. By contrast, marked CD31 mRNA expression was induced only when PDL cells were cultured with both heparin and FGF-2. Western blot analysis showed that the CD31 protein was induced in PDL cells upon treatment with both heparin and FGF-2 for 3 weeks. PDL cells derived from deciduous teeth inducibly express EC-specific markers and thus have the potential to differentiate into a vascular cell lineage.
\end{abstract}

\section{Introduction}

Endothelial cells (ECs), which are derived from vascular progenitor cells, are responsible for angiogenesis and the events of wound healing (1). They are characterized by the expression of vascular endothelial-cadherin (VE-cadherin, CDH5) (2-4), vascular endothelial growth factor receptor 2

Correspondence to: Dr Tomokazu Hasegawa, Department of Pediatric Dentistry, School of Dentistry, Iwate Medical University, 19-1 Uchimaru, Morioka 020-8505, Japan

E-mail: hasegawa@iwate-med.ac.jp

Key words: endothelial cell marker, periodontal ligament, deciduous teeth
(VEGFR2, KDR) (5,6), and CD31 (also called platelet endothelial cell adhesion molecule-1, PECAM-1) (7). Since a blood supply is crucial for wound healing and tissue regeneration, it is important to determine how the progenitors of ECs differentiate into vascular cells in order to establish a practical strategy for regenerative therapy (1).

The periodontal ligament (PDL) is located between the tooth root and alveolar bone (8). Most PDL cells are fibroblasts with relatively high alkaline phosphatase (ALP) activity $(9,10)$. Fibroblasts derived from the PDL have the ability to form bone-like tissues in vitro, similar to osteoblasts $(9,10)$, and thus PDL cells function similarly to osteoblasts in hard tissue formation. Recently, several studies have demonstrated that PDL cells also differentiate into cementoblastic cells and adipogenic cells in vitro $(1,11,12)$. Therefore, the PDL probably contains pluripotent progenitor cells or putative stem cells.

For therapy involving PDL tissue, biologically active soluble factors such as cytokines and growth factors are being evaluated for clinical use in the regeneration of periodontal tissue damaged or lost as a result of periodontitis. Of these factors, basic fibroblast growth factor (FGF-2) is a multifunctional growth factor that has a variety of effects, including the induction of proliferation and differentiation in a wide range of mesodermal and neuro-ectodermal cells. Moreover, FGF-2 is one of the most potent angiogenesis inducers (13). Therefore, we investigated whether FGF-2 induces EC-specific markers in cultured PDL cells in vitro.

In this study, we demonstrated that the expression of VE-cadherin, VEGFR2 and CD31 mRNA is induced in cultured PDL cells by treatment with heparin alone or with FGF-2. We also demonstrated CD31 protein expression in PDL cell cultures using Western blot analysis. This is the first report on the inducible expression of the endothelial cell phenotype by PDL cells derived from human deciduous teeth.

\section{Materials and methods}

Reagents. FGF-2 was obtained from R\&D Systems (Minneapolis, MN, USA). Anti-CD31 monoclonal antibody for the Western blot analysis was obtained from Cell Signaling Technology, Inc. (Danvers, MA, USA). 
Table I. Primers used in the quantitative real-time reverse transcription-polymerase chain reaction (real-time PCR).

\begin{tabular}{|c|c|c|}
\hline Gene name & Primer & Oligonucleotide sequence $\left(5^{\prime}-3^{\prime}\right)$ \\
\hline VE-cadherin & $\begin{array}{l}\text { Forward: } \\
\text { Reverse: }\end{array}$ & $\begin{array}{l}\text { GAGACCTCATCAGCCTTGGGATAG } \\
\text { CTGGATTTGCCAGCATTTGAGA }\end{array}$ \\
\hline VEGFR2 & $\begin{array}{l}\text { Forward: } \\
\text { Reverse: }\end{array}$ & $\begin{array}{l}\text { CCAGGCAACGTAAGTGTTCGAG } \\
\text { GGGACCCACGTCCTAAACAAAG }\end{array}$ \\
\hline CD31 & $\begin{array}{l}\text { Forward: } \\
\text { Reverse: }\end{array}$ & $\begin{array}{l}\text { GACGTGCAGTACACGGAAGTTCA } \\
\text { GTGCATCTGGCCTTGCTGTC }\end{array}$ \\
\hline GAPDH & $\begin{array}{l}\text { Forward: } \\
\text { Reverse: }\end{array}$ & $\begin{array}{l}\text { GCACCGTCAAGGCTGAGAAC } \\
\text { TGGTGAAGACGCCAGTGGA }\end{array}$ \\
\hline
\end{tabular}

VE-cadherin, vascular endothelial cadherin; VEGFR2, vascular endothelial growth factor receptor 2; GAPDH, glyceraldehyde 3-phosphate dehydrogenase.

Cell culture. PDL tissues were obtained from the middle third of the root surfaces of healthy human deciduous teeth (obtained from three donors, aged 7-8 years), as described previously $(14,15)$. Informed consent was obtained from the donors' parents before tooth extraction, which was carried out in our hospital during the course of orthodontic treatment. The study protocol was approved by the Ethics Committee of Iwate Medical University, School of Dentistry (no. 01101).

The PDL tissues were cut into pieces using a surgical blade and were digested with collagenase $(2 \mathrm{mg} / \mathrm{ml})$ at $37^{\circ} \mathrm{C}$ for $30 \mathrm{~min}$. Then, the tissues were washed with Dulbecco's phosphate-buffered saline (PBS), placed on culture dishes, and maintained in $\alpha$-modified minimum essential medium ( $\alpha$-MEM; Gibco BRL, Gaithersburg, MD, USA) supplemented with $10 \%$ fetal bovine serum (FBS; Gibco BRL). Fibroblastic cells that outgrew from the PDL tissues were used as PDL cells. When the cells reached confluence, they were detached with $0.2 \%$ trypsin and $0.02 \%$ EDTA $\cdot 4 \mathrm{Na}$ in PBS, and subcultured at a 1:4 split ratio. The experiments were performed using 4th-passage cells cultured in $\alpha$-MEM supplemented with $10 \%$ FBS in the absence or presence of $15 \mathrm{ng} / \mathrm{ml}$ heparin or $10 \mathrm{ng} / \mathrm{ml} \mathrm{FGF-2} \mathrm{for} 2$ days. The cultures were maintained at $37^{\circ} \mathrm{C}$ in a humidified atmosphere of $5 \% \mathrm{CO}_{2}$ in air.

Isolation of total RNA. Total RNA was extracted from the cultured PDL cells by using Isogen (Nippon Gene, Tokyo, Japan) as described previously $(14,15)$. The pellet of total RNA was washed briefly with $75 \%$ ethanol, resuspended in $30 \mu \mathrm{l}$ of diethylpyrocarbonate (DEPC)-treated water, and stored at $-80^{\circ} \mathrm{C}$. The concentration of total RNA was determined spectrophotometrically by measuring the optical density at $260 \mathrm{~nm}$.

Quantitative real-time reverse transcription-polymerase chain reaction $(P C R)$. The RNA sample $(1 \mu \mathrm{g})$ was reversetranscribed to first-strand cDNA using a PrimeScript RT Reagent Kit (Takara Shuzo, Kyoto, Japan) according to the manufacturer's protocol. A Thermal Cycler Dice Real-time System (Takara Shuzo) was used for the two-step reverse transcription-PCR. The cDNA was amplified with SYBR Premix ExTaq and specific oligonucleotide primers for target sequences encoding parts of VE-cadherin, VEGFR2 and CD31. The primers (listed in Table I) were designed based on the cDNA sequences of human mRNA for VE-cadherin, VEGFR2, CD31 and glyceraldehyde 3-phosphate dehydrogenase (GAPDH). Amplification conditions consisted of $10 \mathrm{sec}$ at $95^{\circ} \mathrm{C}$, followed by 40 cycles at $95^{\circ} \mathrm{C}$ for $5 \mathrm{sec}$ and $60^{\circ} \mathrm{C}$ for $30 \mathrm{sec}$, with a final $15 \mathrm{sec}$ at $95^{\circ} \mathrm{C}$ and $30 \mathrm{sec}$ at $60^{\circ} \mathrm{C}$ in the Thermal Cycler Dice Real-time System.

Western blot analysis of cell surface CD31 expression in PDL cells. After treatment with heparin and/or FGF-2 for 21 days, PDL cells were washed twice with PBS and then treated with lysis buffer [10 mM HEPES-KOH (pH 7.5), $100 \mathrm{mM} \mathrm{KCL}$ and $0.1 \% \mathrm{NP}-40]$. The protein concentration in the cell lysate was measured using a BioRad Protein Assay Kit (BioRad, Hercules, CA, USA). Each sample containing equal amounts of protein was separated by $10 \%$ SDS-polyacrylamide gel electrophoresis (SDS-PAGE) and transferred to a polyvinylidene difluoride membrane (Millipore, Bedford, MA, USA). After being blocked with 5\% skim milk in Tris-buffered saline containing $0.1 \%$ Tween-20 (TBST), the membrane was incubated with mouse anti-human CD31 antibodies and subsequently with anti-mouse secondary antibodies (Zymed Laboratories Inc., San Francisco, CA, USA). Specific protein bands on the membrane were detected by using an enhanced AP Conjugate Substrate Kit (BioRad) as described previously $(14,15)$.

Statistical analysis. The results are expressed as the mean \pm SEM. Statistical significance was determined by one-way analysis of variance Bonferroni comparisons between pairs of groups. Data with a P-value $<0.01$ were considered statistically significant.
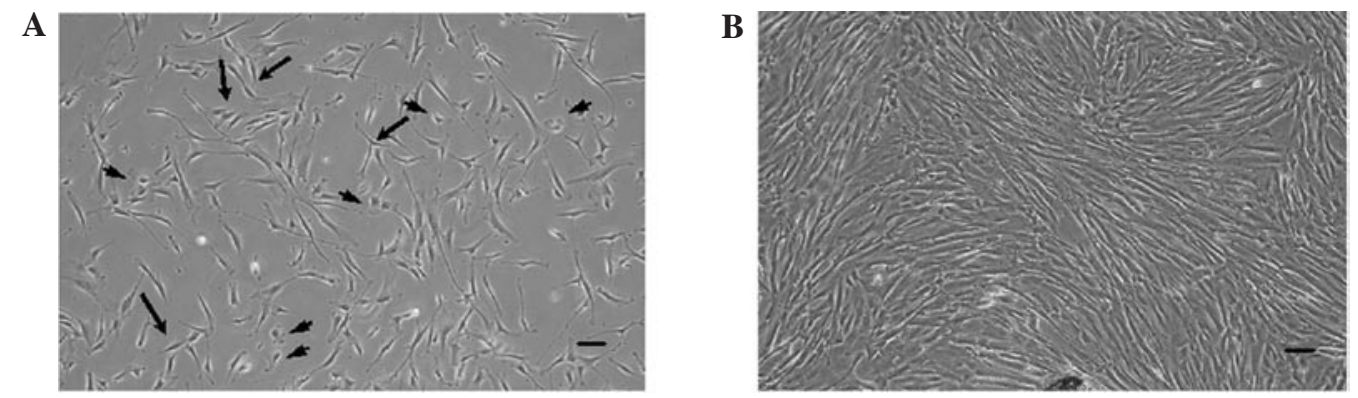

Figure 1. Morphologies of PDL cells derived from deciduous teeth observed by phase-contrast microscopy in primary culture. (A) Most of the PDL cells outgrown from the PDL tissues were fibroblastic cells (arrows). At 10 days after isolation, some cells exhibited a polygonal shape similar to epithelial cells and mature osteoblasts (arrowheads). Few cells exhibited a cell morphology similar to senescent fibroblast-like cells. (B) After reaching confluence and subculture, it was not possible to distinguish between the cell morphologies. Bar, $100 \mu \mathrm{M}$. 
A

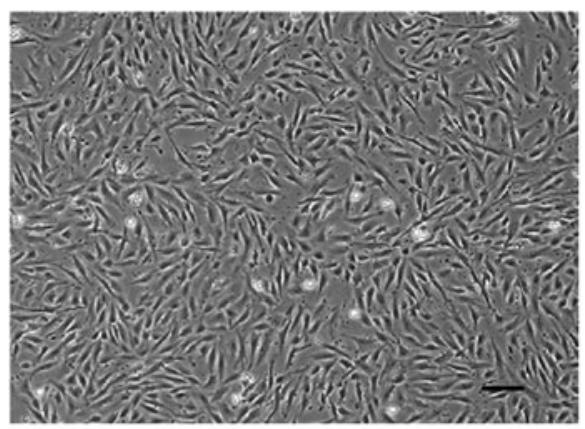

C

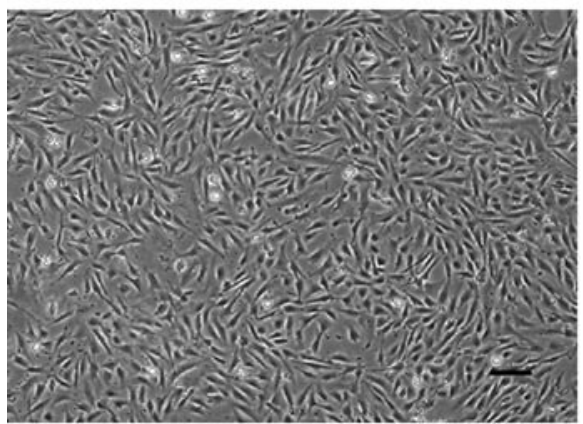

B

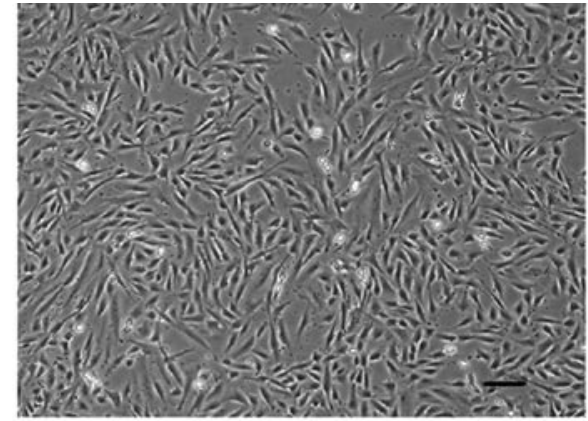

D

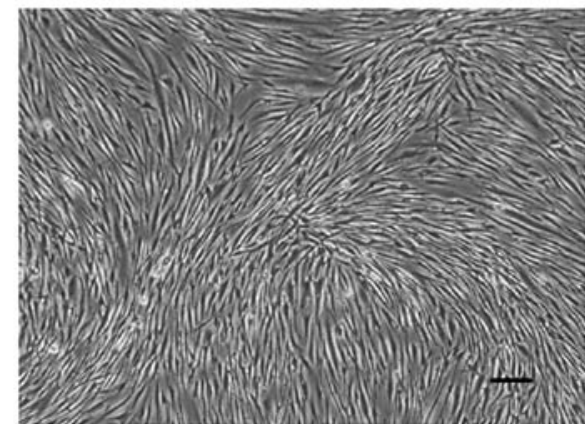

Figure 2. Effect of heparin with/or FGF-2 on the morphologies of PDL cells derived from deciduous teeth. (A) PDL cells were cultured in the absence of FGF-2 for 2 days. (B) PDL cells were cultured in the presence of heparin alone for 2 days. (C) PDL cells were cultured in the presence of FGF-2 alone for 2 days. (D) PDL cells were cultured in the presence of both FGF-2 and heparin for 2 days. The PDL cell morphology changed into a long and thin spindleshaped fibroblastic form. Bar, $100 \mu \mathrm{M}$.

A

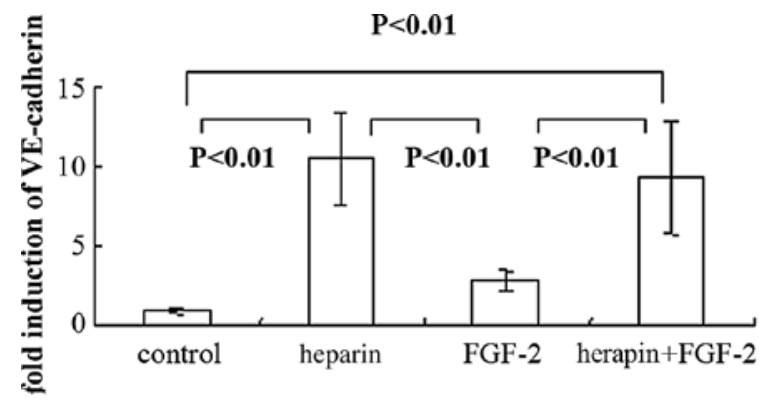

C

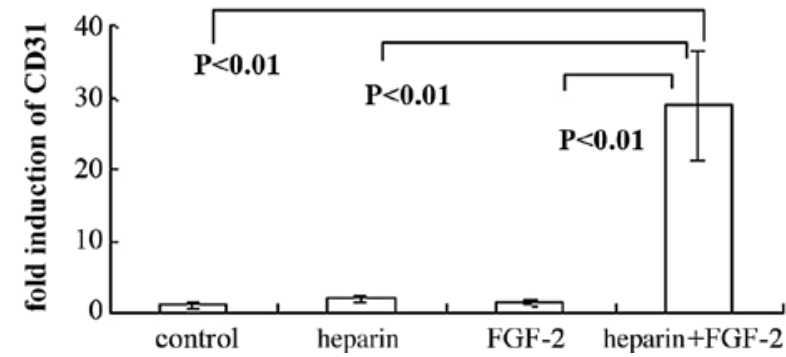

\section{Results}

PDL cells derived from deciduous teeth in primary culture show various cell morphologies. As shown in Fig. 1, PDL cells exhibited various cell types at 10 days after isolation from PDL tissues using phase-contrast microscopy. Most of the PDL cells derived from deciduous teeth were fibroblastic cells (Fig. 1A, arrows). Some cells were polygonal shape, similar to epithelial cells and mature osteoblasts (Fig. 1A, arrowheads). A few cells were senescent fibroblastic cells (Fig. 1A, arrowheads). After
B

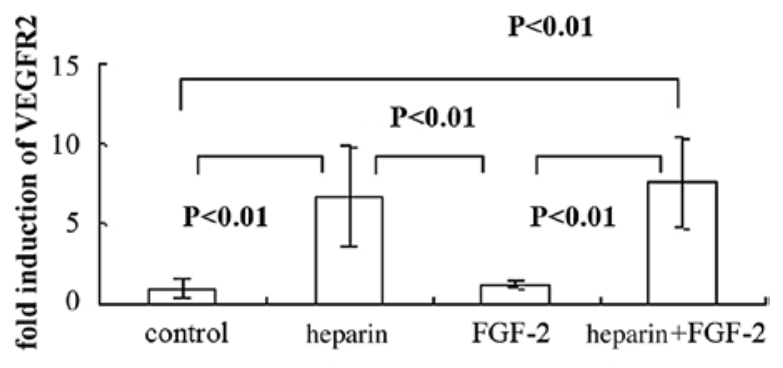

Figure 3. Effect of heparin with or without FGF-2 on endothelial cell marker mRNA expression of PDL cells. (A) VE-cadherin, (B) VEGFR2 and (C) CD31 mRNA expression in the culture of PDL cells. VE-cadherin and VEGFR2 mRNA was markedly increased by the treatment with heparin and both heparin and FGF-2. However, VE-cadherin and VEGFR2 expression did not increase in the presence of FGF-2 alone. Expression of CD31 was significantly induced only in the presence of both heparin and FGF-2 $(n=11)$.

the cells reached confluence and subculture, it was not possibe to distinguish between the cell morphologies (Fig. 1B).

Morphological changes in PDL cells are induced by treatment with both heparin and FGF-2 for 2 days. After culturing for 2 days, PDL cells were subconfluent in the control media and the presence of heparin or FGF-2 (Fig. 2). Upon treatment with both heparin and FGF-2, PDL cells reached confluence, and their morphology was altered into long and thin spindleshaped fibroblasts (Fig. 2D). 
A

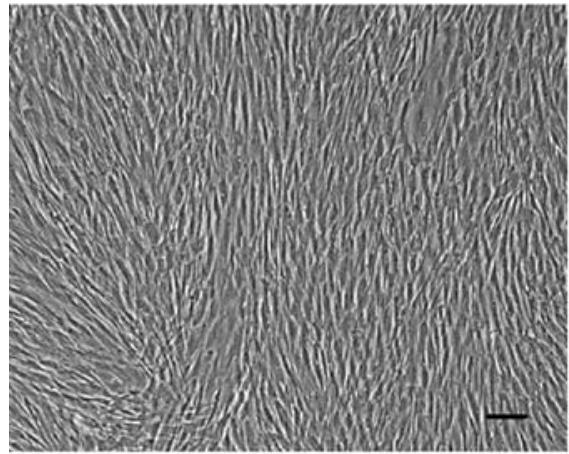

C

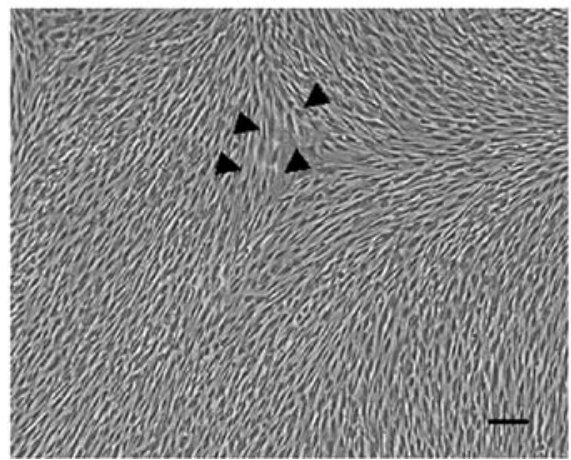

B

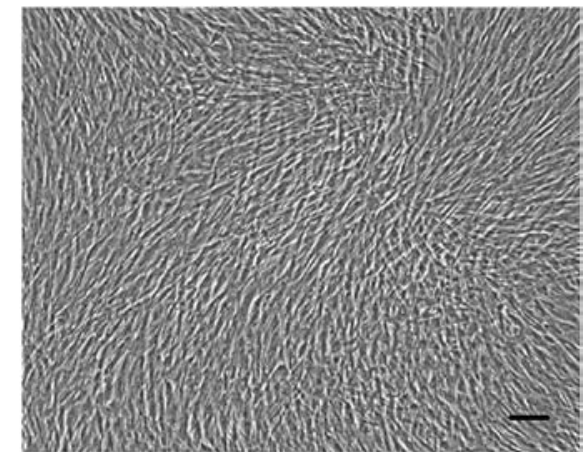

D

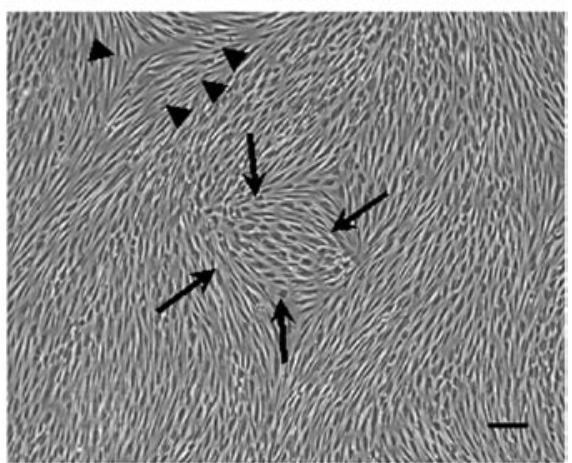

Figure 4. Effect of heparin with/or FGF-2 on the morphologies of PDL cells derived from deciduous teeth. (A) PDL cells were cultured in the absence of FGF-2 for 3 weeks. (B) PDL cells were cultured in the presence of heparin alone for 3 weeks. (C) PDL cells were cultured in the presence of FGF-2 alone for 3 weeks. PDL cells exhibited a long and thin spindle-shaped fibroblastic morphology (arrowheads). (D) PDL cells were cultured in the presence of both heparin and FGF-2 for 3 weeks. PDL cells showed a long and thin spindle-shaped fibroblastic morphology (arrowheads) and polygonal morphology (arrows). Bar, $100 \mu \mathrm{m}$.

A

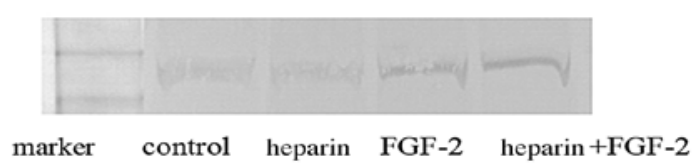

B

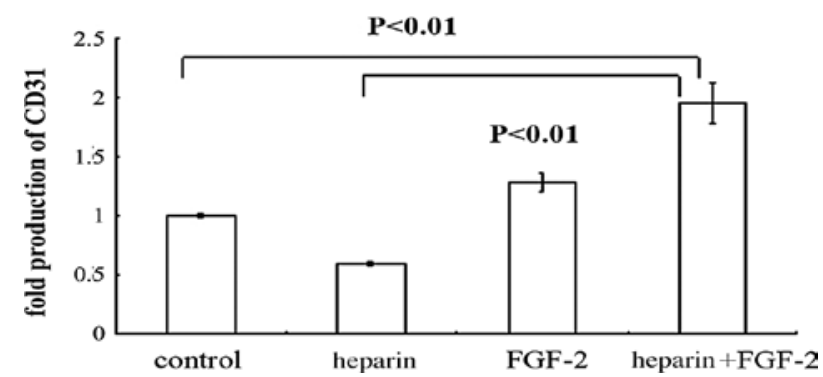

Figure 5. Treatment with heparin and FGF-2 induced CD31 expression in PDL cells. (A) When PDL cells were cultured in control media and heparin only, CD31 expression was weakly detected. Upon treatment with FGF-2 or both heparin and FGF-2, CD31 expression was induced in PDL cells. (B) The ratios of CD31 expression in PDL cells in the control media (control values were normalized to 1). Data are expressed as the mean \pm SEM. CD31 expression was stimulated by treatment with both heparin and FGF-2.

EC-specific markers are induced in PDL cells cultured in the presence of heparin alone or with FGF-2 for 2 days. As shown in Fig. 3A and B, when PDL cells were cultured in the presence of heparin alone, VE-cadherin and VEGFR2 mRNA expression was markedly increased. Treatment with both heparin and FGF-2 also increased both VE-cadherin and VEGFR2 expression in PDL cells (Fig. 3A and B). However, upon treatment with FGF-2 alone, VE-cadherin and VEGFR2 mRNA expression was not induced in cultured PDL cells (Fig. 3A and B). In contrast, CD31 expression was significantly induced by treatment with both heparin and FGF-2 (Fig. 3C), but not with heparin or FGF-2 alone.

Morphological changes in PDL cells are induced by treatment with FGF-2 alone and both heparin and FGF-2 for 3 weeks. As shown in Fig. 4, PDL cells treated with FGF-2 alone and/ or heparin reached confluent multilayers when culturing for 3 weeks. Due to confluence, there were no large differences in the morphology between the control and the cells treated with heparin alone (Fig. 4A and B). Upon culturing in the presence of FGF-2 alone and both heparin and FGF-2, PDL cells showed long and thin spindle-shaped fibroblastic morphology (Fig. 4C and D, arrowheads) and polygonal morphology (Fig. 4D, arrows).

CD31 expression was induced in PDL cells cultured in the presence of FGF-2 and/or heparin. To determine whether CD31 protein was induced in PDL cells, they were cultured in the presence of heparin alone, FGF-2 alone or both heparin and FGF-2 (Fig. 5). Upon treatment with heparin alone or with FGF-2 for 2 days, CD31 expression was not detected by using Western blot analysis (data not shown). Until 2 weeks of culture, CD31 protein expression was not detected in PDL cells. After treatment with FGF-2 alone and both heparin and FGF-2 for 3 weeks, CD31 expression was detected by Western blotting (Fig. 5A). Compared with the control, the production 
of CD31 was induced in PDL cells by treatment with both FGF-2 and heparin (Fig. 5B).

\section{Discussion}

Tissue regeneration and homeostasis in response to pathological and environmental changes such as periodontal disease, wounding and tooth movement with orthodontic treatment are thought to depend in large part upon angiogenesis in the periodontal tissue. PDL cells exist surrounding tooth roots and thus are likely to play an important role in periodontal tissue maintenance. Recently, PDL cells were shown to have biological characteristics in common with bone marrow mesenchymal cells, suggesting that multipotent stem cells are present in the PDL tissue (11). However, it remains unclear whether PDL cells derived from human deciduous teeth can give rise to the endothelial cell (EC) lineage in vitro. To investigate PDL tissue regeneration and homeostasis, it is crucial to determine whether PDL cells have the ability to become ECs.

In this study, we used PDL cells derived from human deciduous teeth to investigate the effects of heparin and FGF-2 on the expression of markers specific for mature ECs: VE-cadherin (2-4), VEGFR2 (5,6) and CD31 (7). Previous studies have found that PDL cells, which are not stimulated with FGF-2, do not express EC markers such as CD31 $(12,16)$. We also found that PDL cells do not express VE-cadherin, VEGFR2, or CD31 without treatment with FGF-2. Surprisingly, PDL cells increased the expression of VE-cadherin and VEGFR2 when cultured in the presence of heparin alone or with FGF-2. The expression of CD31 was also significantly increased in PDL cells cultured with both heparin and FGF-2. This discrepancy might have been due to the presence of heparin in the cell culture conditions. Heparin, a soluble derivative of heparin sulfate and a well known cofactor for FGF-2, substantially enhances the activity of FGF-2 (17). Our observation that the addition of heparin in the absence of FGF-2 stimulated the expression of VE-cadherin and VEGFR2 suggests that heparin enhanced the activity of endogenously produced FGF-2. Nevertheless, other mechanisms cannot be excluded. For example, heparin might have enhanced the activity of growth factors present in the fetal bovine serum, resulting in up-regulated expression of VE-cadherin and VEGFR2 in PDL cells.

Compared with mRNA expression, CD31 protein production showed relatively small changes in the PDL cells. However, the treatment with FGF-2 was sufficient to induce CD31 protein. As shown in Fig. 5, when PDL cells were treated with heparin and FGF-2, CD31 production was increased approximately 2-fold compared with the control group. The amount of protein, which is determined, not only by the mRNA level, but also by multiple processes of protein synthesis and degradation, may be a critical factor.

Here, we demonstrated for the first time that PDL cells derived from human deciduous teeth inducibly express EC-specific markers, such as VE-cadherin, VEGFR2 and CD31 upon treatment with heparin alone or with FGF-2 in vitro. These findings are useful to understand how to regenerate PDL tissue by inducing angiogenesis.

\section{Acknowledgements}

This work was supported, in part, by a Grant-in-Aid for Scientific Research (no. 18592026 to A.I., no. 19791370 to N.C., and no. 18592239 to T.H.) from the Ministry of Education, Culture, Sports, Science, and Technology of Japan; the Open Research Project and High-Tech Research Project from the Ministry of Education, Culture, Sports, Science, and Technology of Japan; the Akiyama Foundation (to T.H., 2005); and a grant from the Keiryokai Research Foundation (no. 100 to N.C., 2008, and no. 106 to T.H., 2009).

\section{References}

1. Bartold PM, Shi S and Gronthos S: Stem cells and periodontal regeneration. Periodontol 2000 40: 164-172, 2006.

2. Dejana E: Endothelial cell-cell junctions: happy together. Nat Rev Mol Cell Biol 5: 261-270, 2004.

3. Cavallaro U, Liebner S and Dejana E: Endothelial cadherins and tumor angiogenesis. Exp Cell Res 312: 659-667, 2006.

4. Carmeliet P, Lampugnani MG, Moons L, et al: Targeted deficiency or cytosolic truncation of VE-cadherin gene in mice impairs VEGF-mediated endothelial survival and angiogenesis. Cell 98: 147-157, 1999.

5. Lampugnani MG, Orsenigo F, Gagliani MC, Tacchetti C and Dejana E: Vascular endothelial cadherin controls VEGFR-2 internalization and signaling from intracellular compartments. J Cell Biol 174: 593-604, 2006.

6. Yamaguchi TP, Dumont DJ, Conlon RA, Breitman ML and Rossant J: flk-1, an flt-related receptor tyrosine kinase, is an early marker for endothelial cell precursors. Development 118: 489-498, 1993.

7. Hristov M, Erl W and Weber PC: Endothelial progenitor cells: mobilization, differentiation and homing. Arterioscler Thromb Vasc Biol 23: 1185-1189, 2003.

8. Freeman, E: Periodontium. In: Oral Histology: Development, Structure and Junction. Ten Cate AR (ed). Mosby, St. Louis, pp276-312, 1994.

9. Groeneveld MC, Everts V and Beertsen W: Alkaline phosphatase activity in the periodontal ligament and gingiva of the rat molar: its relation to cementum formation. J Dent Res 74: 1374-1381, 1995.

10. Beertsen W and van den Bos T: Alkaline phosphatase induces the mineralization of sheets of collagen implanted subcutaneously in the rat. J Clin Invest 89: 1974-1980, 1992.

11. Seo BM, Miura M, Gronthos S, Bartold PM, Batouli S, Brahim J, Young M, Robey PG, Wang C and Shi S: Investigation of multipotent postnatal stem cells from human periodontal ligament. Lancet 364: 149-155, 2004.

12. Nagatomo K, Komaki M, Sekiya I, Sakaguchi Y, Noguchi K, Oda S, Muneta T and Ishikawa I: Stem cell properties of human periodontal ligament cells. J Periodontal Res 41: 303-310, 2006.

13. Ferrara $\mathrm{N}$ and Davis-Smyth $\mathrm{T}$ : The biology of vascular endothelial growth factor (Review). Endocr Rev 18: 4-25, 1997.

14. Hasegawa T, Yoshimura Y, Kikuiri T, Yawaka Y, Takeyama S, Matsumoto A, Oguchi $\mathrm{H}$ and Shirakawa T: Expression of receptor activator of NF-kappa B ligand and osteoprotegerin in culture of human periodontal ligament cells. J Periodont Res 37: 405-411, 2002.

15. Hasegawa T, Kikuiri T, Takeyama S, Yoshimura Y, Mitome M, Oguchi H and Shirakawa T: Human periodontal ligament cells derived from deciduous teeth induce osteoclastogenesis in vitro. Tissue Cell 34: 44-51, 2002.

16. Trubiani O, Isgro A, Zini N, Antonucci I, Aiuti F, Di Primio R, Nanci A, Caputi S and Paganelli R: Functional interleukin-7/ interleukin-7Ralpha, and SDF-1alpha/CXCR4 are expressed by human periodontal ligament derived mesenchymal stem cells. J Cell Physiol 214: 706-713, 2008.

17. Furue MK, Na J, Jackson JP, Okamoto T, Jones M, Baker D, Hata R, Moore HD, Sato JD and Andrews PW: Heparin promotes the growth of human embryonic stem cells in a defined serum-free medium. Proc Natl Acad Sci USA 105: 13409-13414, 2008. 